\title{
Quality of compounded hydrocortisone capsules used in the treatment of children
}

\author{
Uta Neumann1, *, Daniela Burau', *, Sarah Spielmann1, Martin J Whitaker ${ }^{3}$, \\ Richard J Ross ${ }^{3}$, Charlotte Kloft ${ }^{2,+}$ and Oliver Blankenstein ${ }^{1,+}$ \\ ${ }^{1}$ Institute for Experimental Paediatric Endocrinology, Charité Berlin, Berlin, Germany, ${ }^{2}$ Department of Clinical \\ Pharmacy and Biochemistry, Institute of Pharmacy, Freie Universität Berlin, Berlin, Germany, and ${ }^{3}$ Department of \\ Human Metabolism, University of Sheffield, Sheffield, UK \\ *(U Neumann and D Burau contributed equally to this work) \\ ${ }^{\dagger}$ (C Kloft and O Blankenstein are joint senior authors)
}

\author{
Correspondence \\ should be addressed \\ to O Blankenstein \\ Email \\ Oliver.Blankenstein@ \\ charite.de
}

\begin{abstract}
Objectives: Due to the lack of paediatric-licensed formulations, children are often treated with individualized pharmacy-compounded adult medication. An international web-based survey about the types of medication in children with adrenal insufficiency (Al) revealed that the majority of paediatric physicians are using pharmacycompounded medication to treat children with Al. Observations of loss of therapy control in children with congenital adrenal hyperplasia with compounded hydrocortisone capsules and regained control after prescribing a new hydrocortisone batch led to this 'real world' evaluation of pharmacy-compounded paediatric hydrocortisone capsules. Methods: Capsule samples were collected randomly from volunteering parents of treated children suffering from congenital adrenal hyperplasia from all over Germany. Analysis of net mass and hydrocortisone content by highperformance liquid chromatography with ultraviolet (HPLC-UV) detection method was performed based on the European Pharmacopeia.

Results: In a total of 61 batches that were sent, 5 batches could not be analysed because of missing dose information, insufficient number of capsules or were not possible to be evaluated. Fifty-six batches containing 1125 capsules were evaluated. $21.4 \%$ of the batches revealed insufficiency in uniformity of net mass or drug content and additional $3.6 \%$ failed because they did not contain the labelled drug.

Conclusions: Compounded medication is a possible cause of variation of steroid doses in children with adrenal insufficiency or congenital adrenal hyperplasia, putting these vulnerable patients at risk of poor disease control and adrenal crisis. These data may apply to other individualized compounded oral medication as well, emphasizing the need for development of licensed paediatric formulations approved by regulatory authorities.
\end{abstract}

\section{Introduction}

A five-year-old girl with congenital adrenal hyperplasia $(\mathrm{CAH})$ and no history of non-compliance developed accelerated growth and elevated androgens. Visual inspection of her compounded hydrocortisone capsules showed variable capsule filling (Fig. 1); assuming similar fluctuations in hydrocortisone content as in filling, a new batch was prescribed which immediately improved disease control. Based on this observation, we undertook a 'real

(C) 2017 European Society of Endocrinology Published by Bioscientifica Ltd. Printed in Great Britain world' evaluation of pharmacy-compounded paediatric hydrocortisone capsules.

Compounding adult medication is commonly used to provide paediatric doses. An international web-based survey was distributed through the newsletter of the European Society of Paediatric Endocrinology (ESPE) to specify their current treatment of adrenal insufficiency (AI) in neonates and children (1). Sixty-seven participants

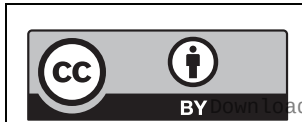

This work is licensed under a Creative Commons Attribution 3.0 Unported License. 


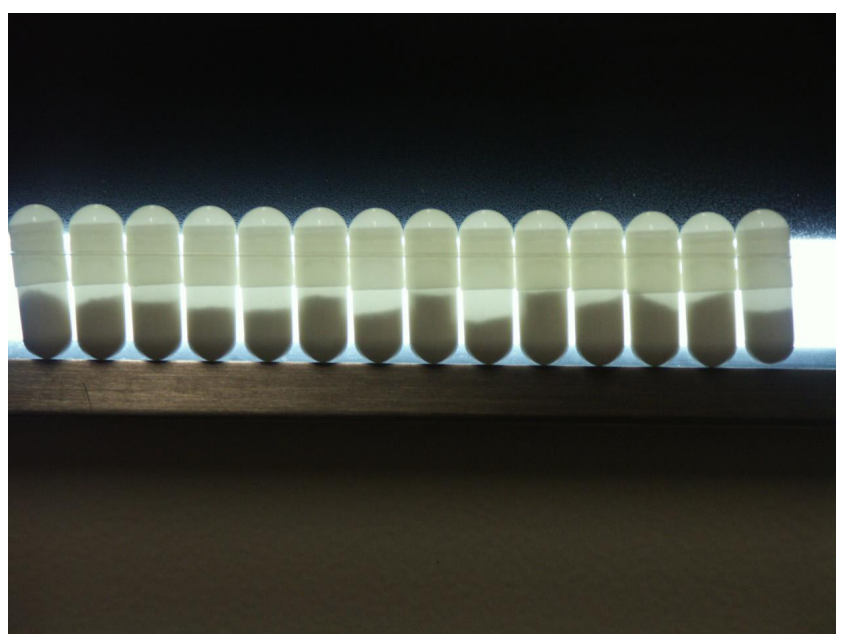

Figure 1

A batch of compounded hydrocortisone capsules dispensed for a child with $\mathrm{CAH}$. Each capsule is meant to contain the same dose, but as can be seen visually, there is great variation in capsule content.

from 16 countries, mostly from Europe, stated the use of divided licensed adult medication (60\%) as off-label and prescription of individualized capsules (55\%) as unlicensed use (1). The prescribed dosage varied from $0.5 \mathrm{mg}$ to $5.0 \mathrm{mg}$ with the vast majority of doses being $1.0 \mathrm{mg}$ and $2.0 \mathrm{mg}$. Compounding is regulated by less stringent criteria than those used for licensed drugs as compounded drugs are neither approved by the EMA (European Medicines Agency) or FDA (Food and Drug Administration) nor required to demonstrate efficacy (2). There is also exemption from Good Manufacturing Practice (GMP) requirements in manufacture of compounded medicines which increases the risks of inaccurate dosing (2). Compounding is by definition an unlicensed use which accounts for up to $60 \%$ of medicines administered to children in Europe and the United States $(3,4)$. The European Pharmacopeia (EP) is a reference work giving common standards for the quality of medicines and their components to provide a basis for the safe use of medicines. The EP contains acceptance criteria for mass and content uniformity to evaluate batches or single capsules (5).

Here, we report a problem with dosing when using compounded hydrocortisone in children suffering from $\mathrm{AI}$ and $\mathrm{CAH}$. These patients require hydrocortisone replacement therapy to avoid life-threatening adrenal crises and careful titration of therapy to control androgen excess and optimize growth and development. The lowest dose available of hydrocortisone in the USA is a $5.0 \mathrm{mg}$ tablet and in Europe a $10.0 \mathrm{mg}$ tablet. However, the average dose used in paediatrics is $2.0 \mathrm{mg}$ with doses as low as $0.5 \mathrm{mg}$ required for neonates and infants (1). There is therefore a necessity to compound hydrocortisone for paediatric use. Use of compounded hydrocortisone medication in children is thereby unlicensed as well as offlabel treatment. Hydrocortisone has a narrow therapeutic index, and patients with CAH have poor health outcomes in adulthood on current glucocorticoid treatment in part related to treatment in childhood $(6,7)$. Poor compliance is often blamed for poor disease control in children with CAH. However, as we report here, this might also be due to the lack of content uniformity in compounded hydrocortisone preparations.

\section{Methods}

A newsletter from the German CAH-patient organisation as well as information provided by treating physicians during regular appointments was used to ask parents and caregivers to participate in this study. Parents or caregivers were invited to send 20 capsules of their child's compounded hydrocortisone medication to our laboratory. The capsules received for this study were prescribed for CAH-patients aged 0-14 years. No information about sex or other clinical variables was collected. Sixty-one batches ( $n_{\text {capsules }}: 1210$, mean 20/batch) from 11 of 16 federal states in Germany from 35 families and produced in 37 different pharmacies were examined. Net mass of capsule content was calculated by weighing each capsule and subtracting the nominal casing mass from the total mass. To calculate uniformity, mean net mass of each batch and deviations from this mean net mass per capsule were calculated. To evaluate the hydrocortisone content, a HPLC-UV method was developed (8) and validated according to EMA Guideline (http://www.ema.europa.eu/docs/ en_GB/document_library/Scientific_guideline/2011/08/ WC500109686.pdf Published 2012. Accessed September 2 , 2013) (9). To calculate uniformity of hydrocortisone content, mean batch content and deviations from these means per capsule were calculated. The results were evaluated based on the EP acceptance criteria for mass and content uniformity (5). A batch failed if $>10 \%$ exceeds tolerable limits and $\geq 1$ capsule exceeds non-tolerable limits (5). The limits used were 10 and 20\% for mass and 15 and $25 \%$ for content. The study was approved by the institutional ethics committee (EA2/134/12). Study participants received a results information letter relating to their supplied batch. 


\section{Results}

Of 61 capsule batches that were received for analysis, 5 batches were excluded from all further analyses - one of them because surprisingly olive oil had been used as excipient in the capsule which leaked out of the capsules and neither net mass nor drug content could be measured; one batch had no dosing information provided, and in three batches, the number of capsules was too small for evaluation. Fifty-six batches containing 1125 capsules were further evaluated in detail, and the results for individual capsules evaluated are shown in Table 1. Two batches contained no hydrocortisone at all but as the primary criteria are based on uniformity analysis of mass and content, these were included in the analysis and passed the content criteria with a homogenous result of 0\% hydrocortisone in all capsules. Batch analysis revealed the following results: for drug content, 49 batches (87.5\%) fulfilled the acceptance criteria based on the EP guidelines, whilst 7 batches (12.5\%) failed. Extreme hydrocortisonecontaining batches showed deviations from mean drug content of $\pm 30-200 \%$. For net mass, 47 batches $(84 \%)$ fulfilled the acceptance criteria, whilst 9 batches (16\%) failed. Thus, of the compounded capsules that contained hydrocortisone, $21.4 \%$ of batches revealed a failure in the acceptance criteria in net mass or drug content: 3 batches with content deviations only (5.4\%), 5 batches with net mass deviation only (9.0\%) and 4 batches with both content and net mass deviation (7.1\%). In summary, $21.4 \%$ failed the uniformity analysis, and 3.6\% did not contain the labelled steroid at all, totalling up to $25 \%$ of batches that cannot ensure safe therapy for the patients.

\section{Discussion}

In our 'real world' analysis, every 4th batch of compounded hydrocortisone revealed a failure of the acceptance criteria based on the EP. In extreme batches that contained hydrocortisone, the drug content deviated from mean drug content by $\pm 30-200 \%$ and some batches

Table 1 Deviations per capsule from either mean net mass or mean drug content.

\begin{tabular}{|c|c|c|}
\hline \multirow{2}{*}{$\frac{\text { Deviation ranges }}{\text { From mean of batch }}$} & \multicolumn{2}{|c|}{$\begin{array}{l}\text { Number }(\%) \text { of capsules per deviation } \\
\text { range with respect to }\end{array}$} \\
\hline & Mean net mass & Mean drug content \\
\hline$< \pm 10 \%$ & $1032(95 \%)$ & $953(84.7 \%)$ \\
\hline$\geq \pm 10 \%$ to $< \pm 20 \%$ & $50(4.6 \%)$ & $102(9.1 \%)$ \\
\hline$\geq \pm 20 \%$ & $3(0.3 \%)$ & $70(6.2 \%)$ \\
\hline
\end{tabular}

did not contain any detectable hydrocortisone, but another glucocorticoid, despite being labelled as such.

Here, we report a problem with dosing when using compounded hydrocortisone in children suffering from $\mathrm{AI}$ and CAH. These patients require hydrocortisone replacement therapy to avoid life-threatening adrenal crises and careful titration of therapy to control androgen excess and optimize growth and development. The lowest dose available for hydrocortisone in the USA is a $5.0 \mathrm{mg}$ tablet and a $10.0 \mathrm{mg}$ tablet in Europe. However, the average dose used in paediatrics is $2.0 \mathrm{mg}$ with doses as low as $0.5 \mathrm{mg}$ required for neonates and infants (1). There is therefore a necessity to compound hydrocortisone for paediatric use. Hydrocortisone has a narrow therapeutic index, and patients with CAH have poor health outcomes in adulthood on current glucocorticoid treatment in part related to treatment in childhood $(6,7)$. Poor compliance is often blamed for poor disease control in children with $\mathrm{CAH}$, and this causes considerable distress to children and parents. However, as we show here, this might also be due to the lack of content uniformity in compounded hydrocortisone.

The advantage of our study is that it was done as a 'real world' analysis of what medication patients receive from their local pharmacy. A limitation of our study is that it was undertaken in only one country, however, the regulations used apply across Europe and are similar to those in USA. Based on our international web-based survey of medication, it is common paediatric practice in most countries to use divided licensed adult medication (60\%) or prescribe individualized capsules (55\%) (1). Sample collection was voluntary and not designed to be representative, thereby we cannot exclude a possible sampling bias because parents in doubt (e.g. receiving oily capsules) of the child's medication might be more likely to participate.

There are no obligatory quality checks of pharmacycompounded medication regarding drug content. Pharmacies may take part in a voluntary external control programme, but these tests are expensive and participation is not monitored or reported. As the regulations for pharmacy-compounded hydrocortisone are identical to those for other pharmacy-produced medication, our findings may also be applicable to other compounded medication. Further studies on a broader range of compounded paediatric medication are needed to evaluate the inherent risk of significant dosing errors caused by e.g. adherence or the pharmaceutic compounding process. This risk may frequently escape 
paediatrician's notice when dealing with therapy failure in paediatric patients.

Our results demonstrate that variations in pharmacycompounded medication might cause poor disease control in children. In newborns and infants, precise dosing of hydrocortisone at low dose is essential; otherwise, these vulnerable patients are at risk of poor disease control and potential adrenal crisis. There is a need for the development of licensed paediatric formulations approved by regulatory authorities such as the EMA and FDA.

Declaration of interest

R J R and M J W are Directors of Diurnal Ltd, UK. The other authors have indicated that they have no potential conflicts of interest to disclose.

Funding

This study was supported by the European Commission Framework 7 grant: TAIN (No: 281654)

\section{Author contribution statement}

U Neumann and S Spielmann conceptualised and designed the study, collected the capsules, coordinated the responses to the parents, carried out initial data analysis, drafted the initial manuscript and approved the final manuscript as submitted. D Burau and C Kloft carried out the capsule analyses as well as the data analysis, drafted the initial manuscript and approved the final manuscript as submitted. M J Whitaker and R J Ross participated in conceptualising the study, drafting and reviewing the manuscript, and approved the final manuscript as submitted. O Blankenstein conceptualised and designed the study, participated in capsule collection, carried out the initial analysis, drafted the initial manuscript and approved the final manuscript as submitted.

\section{Acknowledgements}

We acknowledge and thank all parents and caregivers for providing the hydrocortisone capsules for investigation. We particularly thank the AGS
Patienten-Initiative e.V. for their important cooperation and their active role in recruitment for the study. Charlotte Kloft and Oliver Blankenstein shared senior authorship.

\section{References}

1 Whitaker MJ, Spielmann S, Digweed D, Huatan H, Eckland D, Johnson TN, Tucker G, Krude H, Blankenstein O \& Ross RJ. Development and testing in healthy adults of oral hydrocortisone granules with taste masking for the treatment of neonates and infants with adrenal insufficiency. Journal of Clinical Endocrinology and Metabolism 2015100 1681-1688. (doi:10.1210/jc.2014-4060)

2 Gudeman J, Jozwiakowski M, Chollet J \& Randell M. Potential risks of pharmacy compounding. Drugs in R\&D 201313 1-8. (doi:10.1007/ s40268-013-0005-9)

3 Czarniak P, Bint L, Favie L, Parsons R, Hughes J \& Sunderland B. Clinical setting influences off-label and unlicensed prescribing in a paediatric teaching hospital. PLoS ONE 201510 e0120630. (doi:10.1371/journal.pone.0120630)

4 Conroy S, Choonara I, Impicciatore P, Mohn A, Arnell H, Rane A, Knoeppel C, Seyberth H, Pandolfini C, Raffaelli MP et al. Survey of unlicensed and off label drug use in paediatric wards in European countries. European Network for Drug Investigation in Children. BMJ 2000320 79-82. (doi:10.1136/bmj.320.7227.79)

5 European Pharmacopoeia, 8th ed., 2013.

6 Han TS, Conway GS, Willis DS, Krone N, Rees DA, Stimson RH, Arlt W, Walker BR, Ross RJ \& United Kingdom Congenital Adrenal Hyperplasia Adult Study Executive. Relationship between final height and health outcomes in adults with congenital adrenal hyperplasia: United Kingdom Congenital Adrenal Hyperplasia Adult Study Executive (CaHASE). Journal of Clinical Endocrinology and Metabolism 201499 E1547-E1555. (doi:10.1210/jc.2014-1486)

7 Finkielstain GP, Kim MS, Sinaii N, Nishitani M, Van Ryzin C, Hill SC, Reynolds JC, Hanna RM \& Merke DP. Clinical characteristics of a cohort of 244 patients with congenital adrenal hyperplasia. Journal of Clinical Endocrinology and Metabolism 201297 4429-4438. (doi:10.1210/jc.2012-2102)

8 Kauzor D. HPLC method development to determine hydrocortisone from capsules contributing to a medication safety study. Annual Meeting of the German Pharmaceutical Society: DPhG 2013154.

9 European Medicine Agency. Guideline on Bioanalytical Method Validation. Ed CfMPfHU (CHMP). London: European Medicine Agency (EMA), 2012.

Received 24 March 2017

Revised version received 9 May 2017

Accepted 16 May 2017 